Research Article

\title{
Chemical Fingerprint Profiles and Pharmacodynamic Investigation for Quality Evaluation of Moxa Smoke by UHPLC in a Rat Model of Superficial Infection
}

\author{
Yanling Wang $\left(\mathbb{D},{ }^{1,2}\right.$ Shengbing Wu $\left(\mathbb{D},{ }^{1,2}\right.$ Leijing Chen $\mathbb{D}^{1},{ }^{1}$ Guo Xu $\left(\mathbb{D},{ }^{1,2}\right.$ Xiaoxiao Wang $\left(\mathbb{D},{ }^{1,2}\right.$ \\ Jie Wu $\left(\mathbb{1},{ }^{1,2}\right.$ Bin Wang $\left(\mathbb{1}\right.$, ${ }^{1}$ and Meiqi Zhou $\mathbb{1}^{1,3}$ \\ ${ }^{1}$ Key Laboratory of Xin'an Medicine of the Ministry of Education, Anhui University of Chinese Medicine, Hefei, China \\ ${ }^{2}$ Key Laboratory of Acupuncture and Moxibustion Foundation and Technology of Anhui Province, \\ Anhui University of Chinese Medicine, Hefei, China \\ ${ }^{3}$ Bozhou Institute of Traditional Chinese Medicine, Anhui Academy of Chinese Medicine, Bozhou, China
}

Correspondence should be addressed to Bin Wang; wangbin5654@163.com and Meiqi Zhou; meiqizhou77@sina.com

Received 5 March 2021; Accepted 23 July 2021; Published 2 August 2021

Academic Editor: Fadia S. Youssef

Copyright ( 2021 Yanling Wang et al. This is an open access article distributed under the Creative Commons Attribution License, which permits unrestricted use, distribution, and reproduction in any medium, provided the original work is properly cited.

Introduction. Moxibustion, a traditional Chinese medicine technique, involves the use of moxa smoke from Folium Artemisia argyi to treat various disorders, especially superficial infections. However, there is a higher health risk for people exposed to high levels of moxa smoke for extended durations. Here, we report the first ultra-high-performance liquid chromatography (UHPLC) fingerprint profiles and pharmacodynamic evaluation of moxa smoke, as well as evaluation of its aqueous solution on a rat model of superficial infection. Methods. A novel method for moxa smoke fingerprint profiling was developed using UHPLC under characteristic wavelength. Chromatographic peaks were further analyzed by ultra-high-performance liquid chromatography quadrupole-time-of-flight mass spectrometry (UHPLC-QTOF/MS). 12 sample batches obtained from various Chinese provinces were then analyzed using similarity evaluation, clustering analysis, and principal component analysis. The pharmacodynamics of moxa smoke and moxa aqueous solution were investigated on a rat model of acute skin wound infection. Results. UHPLC fingerprint profiles of 12 batches of moxa smoke were generated at $270 \mathrm{~nm}$ wavelength and 21 chromatographic peaks extracted as common peaks. Similarity between the 12 batches ranged from 0.341 to 0.982 . Based on cluster analysis, the 12 batches of moxa smoke samples were clustered into five groups. Principal component analysis showed that the cumulative contribution of the three principal components reached $90.17 \%$. Eigenvalues of the first, second, and third principal components were 10.794, 6.504, and 1.638 , respectively. The corresponding variance contribution rates were $51.40 \%, 30.97 \%$, and $7.80 \%$, respectively. Pharmacological analysis found that wound healing was slow in the model group relative to the mupirocin ointment, moxa smoke, and aqueous moxa smoke solution groups. Histological analysis revealed markedly reduced tissue inflammation in rats treated with moxa smoke or its aqueous solution. Conclusions. Moxa smoke and its aqueous solution significantly promote wound healing upon superficial infection. A novel quality control method for moxa smoke was established and evaluated for the first time. As its main effects are unchanged, the transformation of moxa smoke into aqueous moxa smoke improves safety and is a simple and controllable process.

\section{Introduction}

Traditional Chinese medicine (TCM) has been widely used in many Asian countries, especially in China, to treat various diseases for millennia [1, 2]. Moxibustion, usually using mugwort from Folium Artemisia argyi has antibacterial and antiseptic effects and is used in TCM for the treatment and prevention of illness through activation of self-healing [3]. Moxibustion with fumigation, also called fumigated moxibustion, is recorded in 52 disease formulae. It has a long history in China with good therapeutic effects against surgical infections [4, 5]. However, during fumigation 
moxibustion, extended exposure to high levels of moxa smoke from mugwort sticks poses a higher health risk [6-10]. Thus, it is necessary to develop effective moxa smoke quality control procedures to ensure its efficacy and safety.

Moxa smoke therapeutic benefits are attributed to numerous active components, which have not been fully elucidated $[11,12]$. Additionally, analysis and identification of moxa smoke active components is challenging due to the diversity and complexity of TCM compounds. TCM fingerprinting is a simple and effective method of evaluating TCM quality and allows a comprehensive detection of a sample's main components $[13,14]$. Coupling UHPLC fingerprints with the Mass Spectrometer (MS) technique allows the evaluation of the complex composition of TCM.

We previously developed a Gas Chromatography coupled with Mass Spectrometer (GC-MS) method for qualitative identification of volatile oils and burnt smoke in Artemisia argyi, which allowed preliminary quality evaluation of moxa smoke [15]. In 2016, we invented a new equipment of collecting moxa smoke, which has been patented by the national intellectual property administration of the People's Republic of China (patent no. ZL 201610105632.3). We recently found that Artemisia argyi volatile oils have good antibacterial effects and more recently, progress in Artemisia argyi genomics has been made $[16,17]$. Here, we report a fingerprint method based on UHPLC and MS for comprehensively evaluating moxa smoke quality [18-21]. Converting moxa smoke into an aqueous form will lower the risk posed by exposing patients to moxa smoke. Moxa smoke fingerprint profiles were constructed, and the common peak identified by UHPLC and MS was selected. Finally, the therapeutic effect of moxa smoke and aqueous moxa smoke on superficial infections was evaluated using animal experiments.

\section{Experiments}

2.1. Materials and Reagents. The 12 batches of mugwort stick $\left(\mathrm{S}_{1}-\mathrm{S}_{12}\right)$ were purchased from Nanyang Yilejia Mugwort Products Co., LTD. (batch no: 20200102), Nanyang Zhanfang Artemisia argyi Co., LTD. (batch no: 20190102), Nanyang Hanyi Trading Co., LTD. (batch no: 20200415), Nanyang Hanai Mugwort Products Co., LTD. (batch no: 20191205), Pingli Zhonghuang Wild Mugwort Scientific Research Industry and Trade Co., LTD. (batch no: 20191205), Qingdao Zhenaitang Traditional Chinese Medicine Technology Co., LTD. (batch no: 20180311), Qichun Haofan Qi Ai Products Co., LTD. (batch no: 20200403), Jiangsu Kangmei Pharmaceutical Co., LTD. (batch no: 20190916), Anhui Lvyingtang Cailong Mugwort Pharmaceutical Co., LTD. (batch no: 1200202), Linxiang Huxiang Ai Biotechnology Co., LTD. (batch no: 20181201), Yantai Aixin Medical Equipment Co., LTD. (batch no: 20200301), and Ningxia Ze Aitang Biotechnology Co., LTD. (batch no: 20200504), respectively. Chromatographic methanol and acetonitrile were purchased from MERCK. Deionized water was prepared using a Milli-Q purification system (Millipore). Ulai was purchased from Shanghai yuanye BioTechnology Co., Ltd. Ammonium acetate and sodium sulfide, including nine hydrate, were analytically pure. The isoflurane was obtained from RWD Life Science Co., Ltd. Mupirocin ointment was purchased from Hubei Renfu Chengtian Pharmaceutical Co., LTD. (batch no: 20200202).

\subsection{Animals and Experimental Design}

2.2.1. Animals. Ethical approval for animal use was granted by the Committee on the Ethics of Animal Experiments of Anhui University of Chinese medicine. Specific Pathogen Free (SPF) grade, male Sprague Dawley (SD) rats (200-250 g) were purchased from the breeding center of Anhui Medical University (License No. SCXK (Lu) 20190003). Animals were housed individually at $18-22^{\circ} \mathrm{C}$, $40-60 \%$ humidity, and $12 \mathrm{~h}$ light/dark cycle with ad libitum access to food and water and minimal environmental noise. All efforts were made to minimize animal suffering in accordance with the principles of the Animal Care and Use Committee, Anhui University of Chinese Medicine.

2.2.2. Bacterial Strain. Staphylococcus aureus was obtained from the Laboratory of Microbiology and Biochemistry, College of Integrated Traditional Chinese and Western Medicine, Shaoquanhu Campus, Anhui University of Chinese Medicine.

2.2.3. Animal Groups. All rats were randomly split into five groups, namely, the normal group, model group, mupirocin ointment group, moxa smoke group, and aqueous moxa smoke group $(n=7)$. A model of skin wound infection was established in all rats except the normal group according to the work in [22]. Briefly, rats were weighed and placed in induction boxes and anesthetized. They were then transferred to an induction box and placed on fixation plates for operation. Next, a large area of primary hair was removed from the back using a hair pusher, followed by complete hair removal with $10 \%$ sodium sulfide nine hydrate. The hairremoved section was then rinsed with normal saline and dried. After hair loss, rats were placed on a treatment table with the back facing up and the back skin disinfected with iodine volts. A $1.5 \mathrm{~cm}$ (diameter) incision was then made in the middle of the spine, and tweezers were used to injure the skin at the incision until the muscle layer was destroyed. The wound was then swabbed with a cotton swab and $80 \mu \mathrm{L}$ $\left(2.53 \times 10^{9}\right)$ of Staphylococcus aureus solution was introduced into the wound. A sterile application was then used to close the wound, ensuring contact between the bacteria solution and skin wound. After waking from anesthesia, rats were placed in a feeding cage with independent ventilation. After $48 \mathrm{~h}$, a significant pyogenic formation was observed on the backs, indicating successful establishment of a rat model of skin wound infection. Model group rats received an equal amount of solvent.

2.2.4. Administration Method. The normal and model group received saline debridement, respectively. The mupirocin ointment group was daubed with $0.30 \mathrm{~mL} / 100 \mathrm{~g}$ of 
mupirocin ointment. The moxa smoke group was daubed with $0.30 \mathrm{mg} / 100 \mathrm{~g}, 0.45 \mathrm{mg} / 100 \mathrm{~g}$, and $0.6 \mathrm{mg} / 100 \mathrm{~g}$ of moxa smoke solution. The administration method was carried out once daily for 7 days in a row. Changes in diet, body weight, and wound size were observed during the modeling period.

2.2.5. Pharmacodynamic Evaluation. On the $16^{\text {th }}$ day, all rats were deeply anesthetized with isoflurane $(3 \mathrm{~mL} / \mathrm{kg})$. Body weight, diet, and wound healing were monitored during the 7-day treatment. Wound tissues were obtained and fixed in $10 \%$ neutral formalin, and histological analysis conducted using Hematoxylin-Eosin (H\&E) staining.

\subsection{Analysis and Identification for Moxa Smoke}

2.3.1. Moxa Smoke Solutions. One mugwort stick (about $20 \mathrm{~g}$ ) from the Henan region, China, was burnt to generate moxa smoke. An atomizer with $20 \mathrm{~mL}$ deionized water was used to atomize incessantly at a speed of $3 \mathrm{~mL} / \mathrm{min}$ to provide fogdrop (particle diameter $=3.9 \mu \mathrm{m}$ ). Moxa smoke and fogdrop were mixed in a $3 \mathrm{~L}$ container. The whole process takes about $40 \mathrm{~min}$. Aqueous moxa smoke solution $\mathrm{S}_{1}$ was then prepared in a $25 \mathrm{~mL}$ volumetric flask. Aqueous moxa smoke solutions for the other 11 sample solutions $\left(\mathrm{S}_{2}-\mathrm{S}_{12}\right)$ were prepared in the same way. The solution was then filtered on a $0.22 \mu \mathrm{m}$ microporous membrane, and the solutions were stored at $4^{\circ} \mathrm{C}$ until analysis. 50 and $75 \%$ aqueous moxa smoke solutions were then prepared by diluting solutions $S_{2}-S_{12}$ with distilled water.

\subsubsection{Selection of Ultraviolet (UV) Absorption Wavelength of} Moxa Smoke Solutions. Objectively setting the UV detection wavelength is very important in UHPLC fingerprints analysis. So, we analyzed all 12 sample batches at full wavelength by scanning with a UV spectrophotometer and recording their absorbance data.

2.3.3. UHPLC-UV Method. All analyses were conducted on an Agilent Eclipse Plus $\mathrm{C}_{8}(2.1 \times 100 \mathrm{~mm}, 1.8 \mu \mathrm{m})$ chromatographic column at column and sample chamber temperatures of $30^{\circ} \mathrm{C}$ and $10^{\circ} \mathrm{C}$, respectively. UV wavelength was set at $270 \mathrm{~nm}$. UHPLC mobile phase was comprised of A (water containing $0.1 \%$ ammonium acetate solution) and $\mathrm{B}$ (acetonitrile). The gradient was set as follows: $0-8 \mathrm{~min}, 99 \%$ A; 8-9 $\min , 99 \%-97 \%$ A; 9-12 $\min , 97 \%$ A; 12-13 min, 97\%95\% A; 13-20 min, 95\% A; 20-21 min, 95\%-90\% A; 21-26 min, 90\% A; 26-32 min, $90 \%-60 \%$ A; $32-33 \mathrm{~min}$, 60\%-30\% A; 33-35 min, 30\% A; 35-36 min, 30\%-99\% A; and $36-40 \mathrm{~min}, 99 \% \mathrm{~A}$. Optimal chromatographic results were obtained at a flow rate of $0.2 \mathrm{~mL} / \mathrm{min}$ and a sample injection volume of $1.0 \mu \mathrm{L}$.

2.3.4. UHPLC-QTOF/MS Method. For UHPLC-QTOF/MS analysis, an ACQUITY I-Class UHPLC equipment coupled with a Xevo G2-XS QTOF/MS detector (Waters Corp.) via an electrospray interface was used. Mass spectrometry was performed in positive and negative ionization modes, and corresponding data were obtained between 200 and $600 \mathrm{~m} / \mathrm{z}$ at a data acquisition rate of $0.5 \mathrm{~s}$. For accuracy, $\mathrm{m} / \mathrm{z}$ values of all ions acquired in the QTOF/MS were real-time adjusted by Lock Spray, with leucine-enkephalin used as the lock mass compound for the positive ionization mode $\left([\mathrm{M}+\mathrm{H}]^{+}\right.$: $\mathrm{m} / \mathrm{z} 556.2771)$ and negative ionization mode $\left([\mathrm{M}-\mathrm{H}]^{-}: \mathrm{m} / \mathrm{z}\right.$ 554.2615). Components of all analyzed samples generally produced molecular adduct ions such as $[\mathrm{M}+\mathrm{H}]^{+}$or $[\mathrm{M}-\mathrm{H}]^{-}$, which were further fragmented by collision energy application.

\section{Results and Discussion}

3.1. Collection Method of Moxa Smoke. 12 batches of mugwort stick from 9 different Chinese regions were burnt to generate moxa smoke. Next, moxa smoke aqueous solution was obtained by mixing the moxa smoke with fogdrop from an atomizer (Figure 1). The use of aqueous moxa smoke may avoid the risk posed to the patient by exposure to moxa smoke.

\subsection{Pharmacodynamic Evaluation}

3.2.1. Monitoring Wound Healing. The flowchart is shown in Figure 2(a). The rat model of skin wound infection was established in 7 days. In the $9^{\text {th }}$ to $12^{\text {th }}$ days, animals in the normal group gained weight steadily. Animals in the model group significantly lost weight in this period and had loose stools. Their wounds did not heal naturally. In contrast, rats in the treated groups, including mupirocin ointment, moxa smoke, and aqueous moxa solution groups, slightly lost weight and had loose stools. On the $13^{\text {th }}$ day, the rats' diet in the treated group gradually improved and weight rose gradually. On the $16^{\text {th }}$ day, the wound areas had significantly reduced before and after treatment (Figures 2(b)-2(c)), showing the moxa smoke could promote the wound healing. Relative to the moxa smoke group, the aqueous moxa smoke group exhibited similar effects.

3.2.2. Pathological Tissue Section Changes. After 7 days of treatment, H\&E pathological analysis (Figure 2(d)) did not uncover inflammatory cell infiltration and revealed that skin epithelial cells were arranged neatly. The wound in the model group was moist, with more exudate and less obvious contraction. Pathological analysis showed no granulation tissue growth. However, a large number of leukocytes, eosinophils, neutrophils, and other inflammatory cells infiltrated the superficial, deep, and subcutaneous skin tissues.

3.3. Establishment of UHPLC Fingerprint of Moxa Smoke. Here, chemical fingerprinting was used, and it is an important technique for the quality evaluation of natural products [23]. Additionally, the identification of the chemical fingerprint of TCMs involves a variety of extraction methods, for example, sonication, reflux, presoaking, pretreatment, and hydrolysis [24]. The fingerprint profiles of 12 batches of moxa smoke (extracted using the pretreatment) were established through using chemometric methods. 


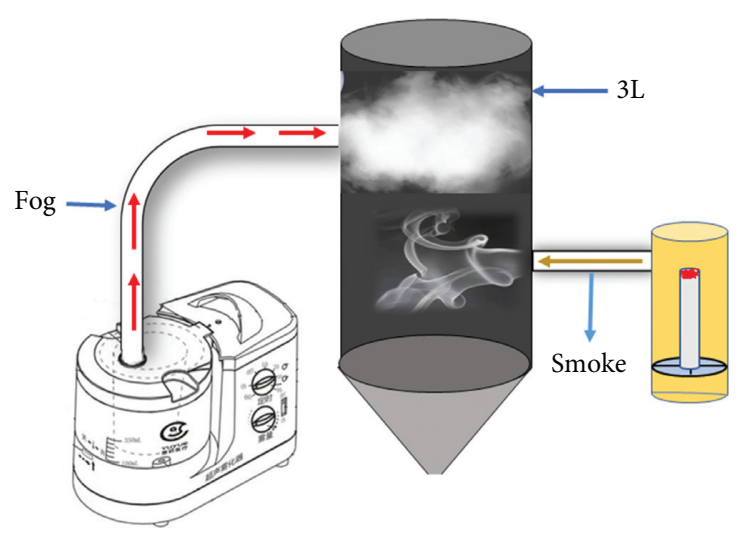

FIgURE 1: Collected equipment of the moxa smoke.

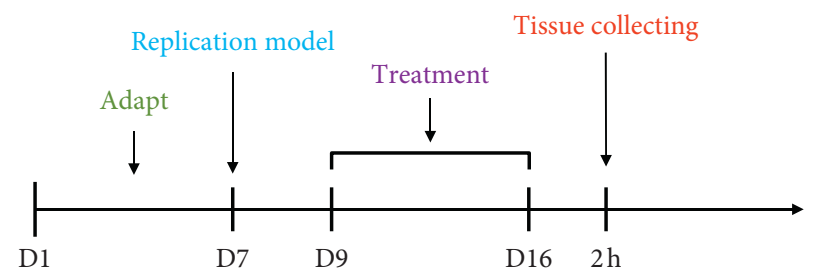

(a)
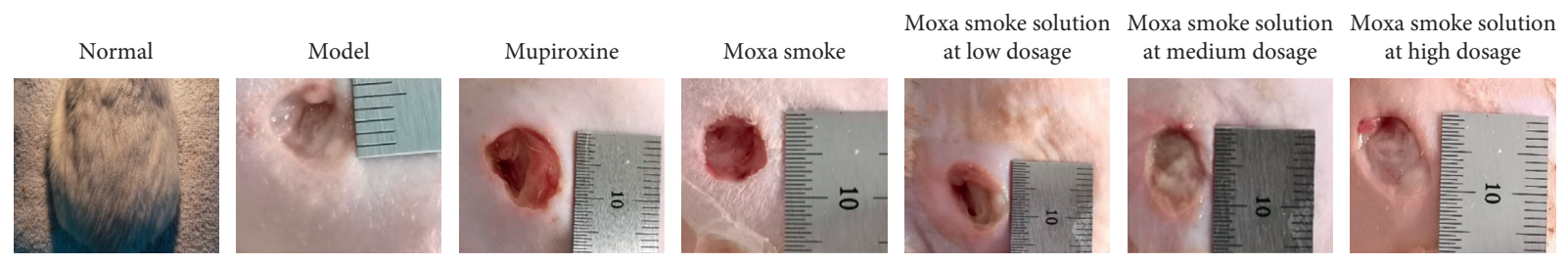

(b)
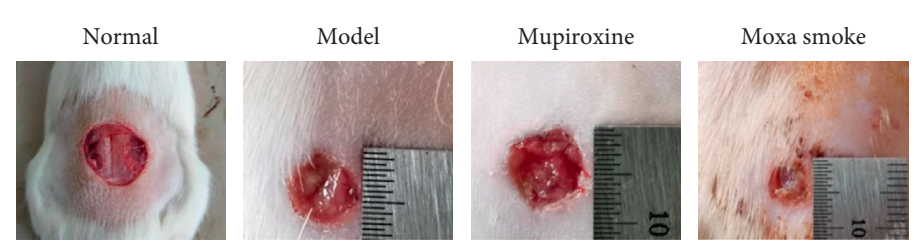

Moxa smoke solution Moxa smoke solution Moxa smoke solution at low dosage

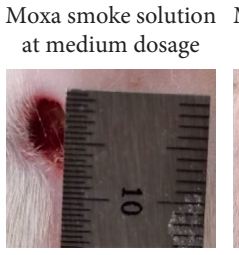
at high dosage

(c)
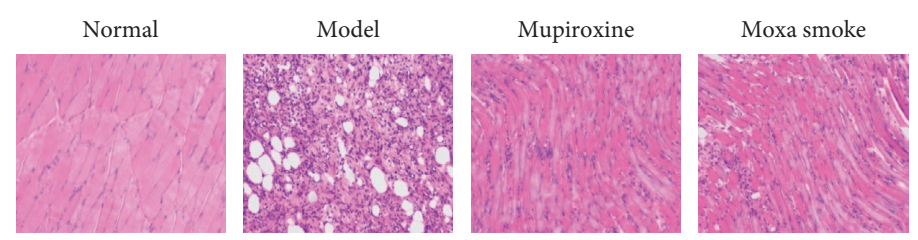

Moxa smoke solution Moxa smoke solution Moxa smoke solution at low dosage

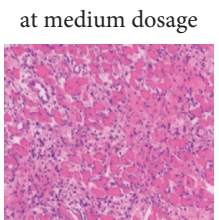

at high dosage

(d)

FIgURE 2: The evaluation of pharmacodynamics experiments.

Specifically, because full wavelength scanning revealed the maximum absorption wavelength for moxa smoke solution as $270 \mathrm{~nm}$ (Figure 3), we set UHPLC detection wavelength at $270 \mathrm{~nm}$. Samples $S_{1}-S_{12}$ were analyzed using the optimal conditions outlined in Section 2.3.3, and data were analyzed using similarity evaluation software of Chinese medicine chromatogram fingerprint (2012 Version) using the sample $S_{1}$ reference map. The median method was used, and time window was set at $0.1 \mathrm{~min}$. Next, chromatogram peaks were analyzed by automatic matching after multipoint correction, and finally, the moxa smoke UHPLC fingerprint was established (Figure 4). 


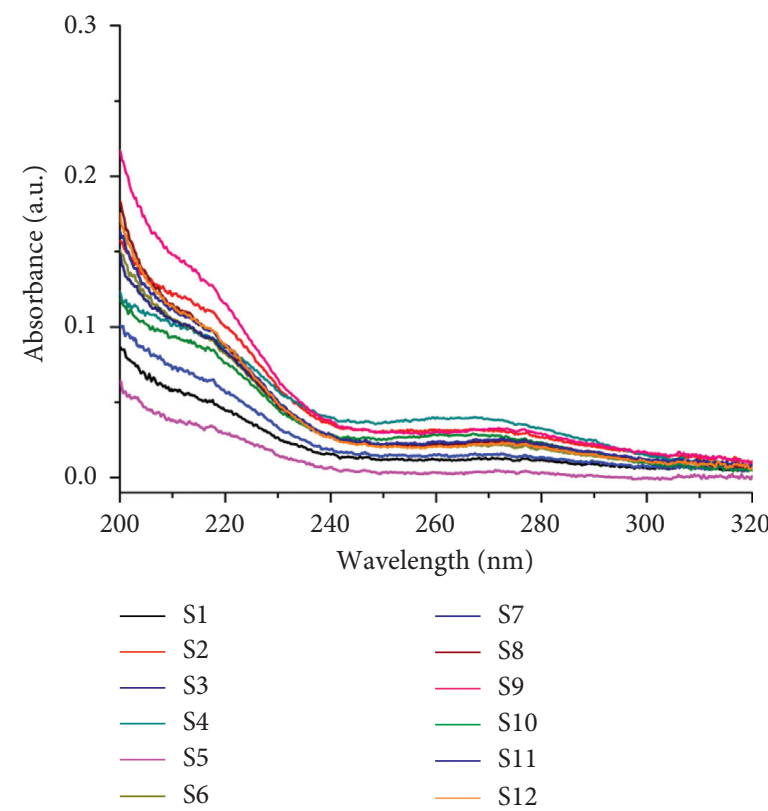

Figure 3: The UV absorption spectrum of 12 batches of moxa smoke solutions.

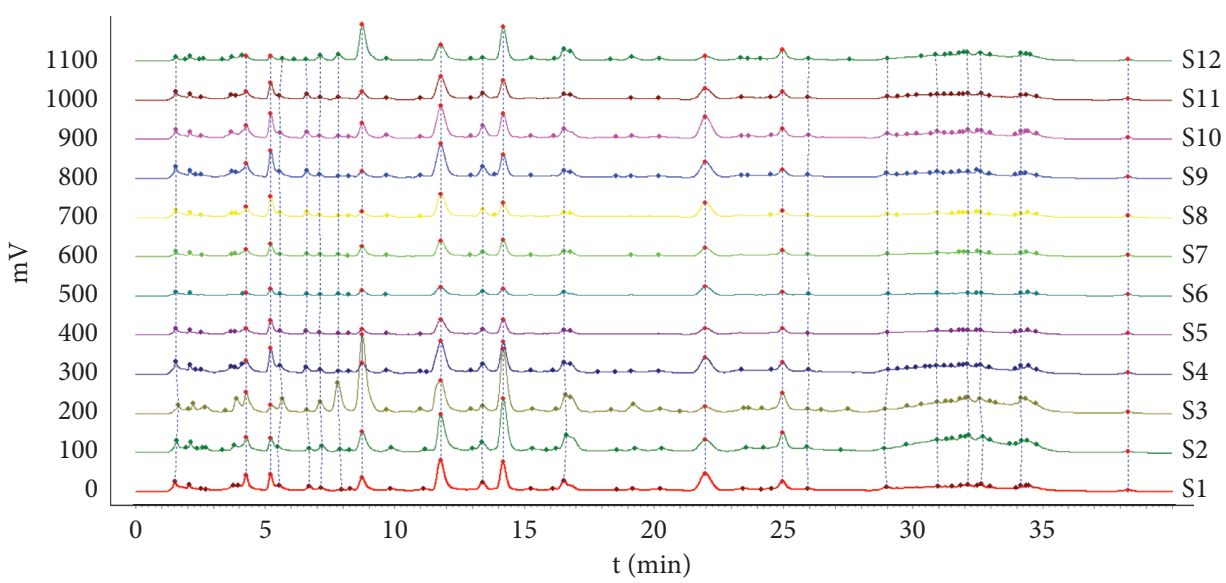

FIgURE 4: The UHPLC fingerprints of moxa smoke solutions at $270 \mathrm{~nm}$.

3.3.1. Investigation of the Fingerprint Methodology. (1) Precision test: $1.0 \mu \mathrm{L}$ sample $S_{1}$ was injected 6 times, and resulting chromatograms were recorded. Relative retention time and relative peak area of each common peak were then investigated. Taking peak $\mathbf{1 2}$ as the reference peak, this analysis showed the RSD of the relative retention time and relative peak area were $<1.7$ and $<3.8 \%$, respectively, indicating good UHPLC precision.

(2) Stability test: $1.0 \mu \mathrm{L}$ of sample $\boldsymbol{S}_{\mathbf{1}}$ was injected at $0,2,6,4$, $8,10,12$, and $24 \mathrm{~h}$, and chromatograms were recorded. Relative retention time and relative peak area for each common peak were then investigated. Taking peak $\mathbf{1 2}$ as the reference peak, this analysis showed that the RSD of the relative retention time and the relative peak area was $<2.0$ and $<1.35 \%$, respectively, indicating good stability for sample $S_{1}$ at $24 \mathrm{~h}$.

(3) Repeatability test: $1.0 \mu \mathrm{L}$ of sample $S_{1}$ was injected 6 times, and chromatograms were recorded. Relative retention time and relative peak area for each common peak were then investigated. Taking peak 12 was taken as the reference peak, and the results showed the RSD of the relative retention time and the relative peak area was $<0.6$ and $<3.6 \%$, respectively, indicating the method's repeatability was fine.

3.3.2. Selection and Identification of Common Peaks. Because the UHPLC chromatogram of the 12 batches of moxa smoke solutions showed that the area of peak $\mathbf{1 2}$ was moderate and its retention was stable, it was selected as the reference peak. Next, the common pattern diagram of moxa smoke fingerprint chromatograms at $270 \mathrm{~nm}$ was exported from the similarity evaluation software (Figure 5). Data of the common peaks of 12 batches of moxa smoke decoction at $270 \mathrm{~nm}$ were also exported from the software. The results showed 33 chromatographic peaks were found in fingerprint 


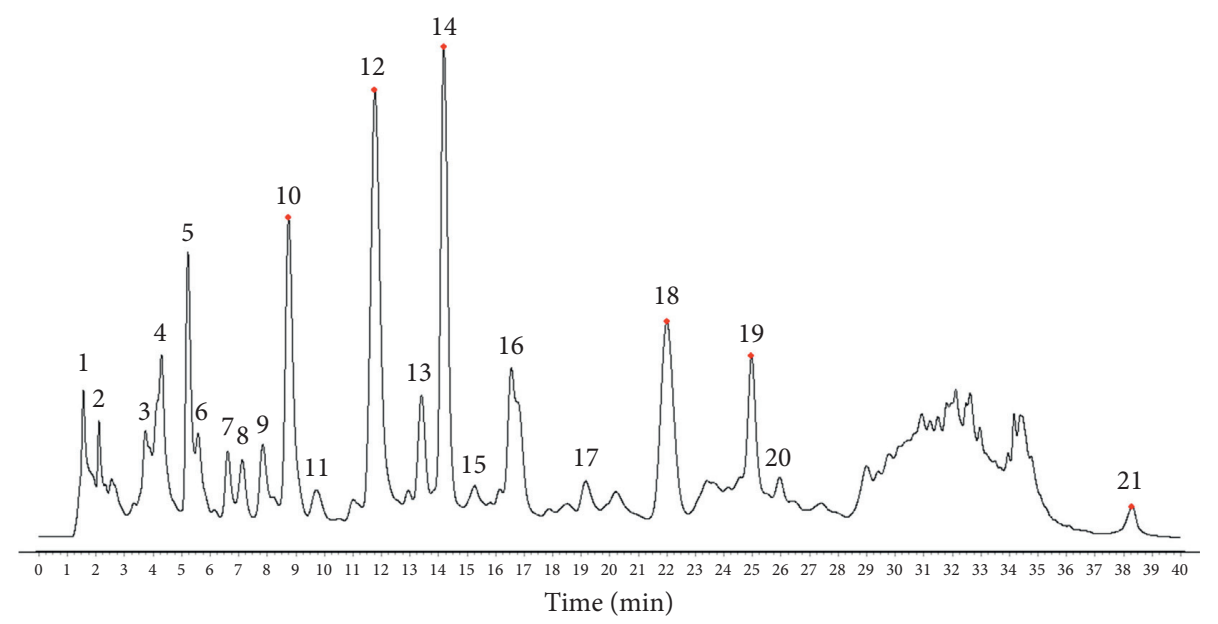

FIgURE 5: The common pattern diagram of moxa smoke fingerprints at $270 \mathrm{~nm}$.

chromatograms and 21 peaks could be matched as common peaks with relative retention times $<1.21 \%$.

QTOF/MS spectra were detected in both positive and negative ion modes. Components in the moxa smoke sample generally produced molecular adduct ions, such as $[\mathrm{M}+\mathrm{H}]^{+}$ or $[\mathrm{M}-\mathrm{H}]^{-}$, which were then fragmented using collision energy. Based on the past study sand fragmentation behavior, common peaks were identified by QTOF/MS spectra. Peaks 2, 5, 7, 8, 9, 10, 12, 14, 18, and 19 were ascribed to azulene (2), furfuryl alcohol (5), cineole (7), 3,5-dimethyl-2cyclohexen-1-one (8), $\alpha$-thujone (9), o-cresol (10), catechol (12), eugenol (14), 4-ethylphenol (18), and 1,2-benzenediol,5-(1,1-dimethylethy)-3-methyl-(19) (Figure

(Table 1).

As well known, phenols generally exhibit antioxidant activity. In this study, we found that there are some phenolic compounds in the combustion of Artemisia argyi, for instance, o-cresol (peak 10), catechol (peak 12), eugenol (peak 14), and 4-ethylphenol (peak 18). So, they may be closely related to the substance basis of the efficacy of moxibustion in the treatment of superficial infectious diseases.

3.3.3. Evaluation of Similarity. Similarity results of 12 moxa smoke solution batches at $270 \mathrm{~nm}$ were obtained from the Similarity Evaluation System of Traditional Chinese medicine chromatographic fingerprint (2012 version) and ranged from 0.341 to 982 (Table 2). Except for the $\boldsymbol{S}_{\mathbf{2}}, \mathbf{S}_{\mathbf{3}}, \mathbf{S}_{\mathbf{4}}$, and $\mathbf{S}_{\mathbf{1 2}}$ samples, sample similarities were $>0.9$.

3.3.4. Clustering Analysis. With the peak areas of 21 common peaks as the standard, Statistical Product and Service Solutions (SPSS) 23.0 software was used, and the method of intergroup mean association was adopted. The included angle cosine was selected by the distance formula of sample similarity to systematically cluster the 12 batches of moxa smoke samples (Figure 7).

Based on cluster analysis, the 12 moxa smoke sample batches were clustered into 5 groups with $\mathbf{S}_{3}, \mathbf{S}_{\mathbf{4}}, \mathbf{S}_{\mathbf{5}}, \mathbf{S}_{\mathbf{6}}$, and $\mathbf{S}_{\mathbf{9}}$ clustered into class I, $\mathbf{S}_{\mathbf{2}}, \mathbf{S}_{\mathbf{7}}, \mathbf{S}_{\mathbf{8}}$, and $\mathbf{S}_{\mathbf{1 2}}$ clustered into class
II, $\mathbf{S}_{\mathbf{1 0}}$ clustered into class III, $\mathbf{S}_{\mathbf{1 1}}$ clustered into class IV, and $\mathbf{S}_{1}$ clustered into class $\mathrm{V}$.

3.3.5. Principal Component Analysis (PCA). SPSS 23.0 was used to standardize the original data, and the characteristic root, and the contribution rate of principal components was used as the basis for selecting principal components for principal component analysis. The top 3 principal components whose eigenvalues were $>1$ were selected for principal component analysis on 21 common peaks (variables) of 12 samples (observed objects). This analysis showed that the cumulative contribution of the 3 principal components reached $90.17 \%$. The eigenvalues of the $1^{\text {st }}, 2^{\text {nd }}$, and $3^{\text {rd }}$ principal components were $10.794,6.504$, and 1.638 , respectively. The corresponding variance contribution rates were $51.40 \%, 30.97 \%$, and $7.80 \%$, respectively. The coordinate system was established with the $1^{\text {st }}, 2^{\text {nd }}$, and $3^{\text {rd }}$ principal components, respectively, and PCA 3D maps for all samples were obtained after projection (Figure 8). Thus, the 21 common peaks can be divided into 3 categories.

After analysis, we obtained the results of the common factor load matrix after rotation (Table 3), indicating that each load amount represents the correlation coefficient between the principal component and corresponding variable. The component model was then obtained on SPSS 23.0 as follows:

$$
\begin{aligned}
& \mathrm{A}_{1}=0.007 \mathrm{~F}_{1}+0.065 \mathrm{~F}_{2}-0.017 \mathrm{~F}_{3}+0.070 \mathrm{~F}_{4}-0.032 \mathrm{~F}_{5}+ \\
& 0.089 \mathrm{~F}_{6}+0.072 \mathrm{~F}_{7}+0.079 \mathrm{~F}_{8}+0.059 \mathrm{~F}_{9}+0.018 \mathrm{~F}_{10}+ \\
& 0.091 \mathrm{~F}_{11}+0.074 \mathrm{~F}_{12}-0.015 \mathrm{~F}_{13}+0.090 \mathrm{~F}_{14}+0.040 \mathrm{~F}_{15}- \\
& 0.037 \mathrm{~F}_{16}+0.065 \mathrm{~F}_{17}+0.086 \mathrm{~F}_{18}+0.088 \mathrm{~F}_{19}+0.088 \mathrm{~F}_{20}- \\
& 0.085 \mathrm{~F}_{21} \\
& \mathrm{~A}_{2}=0.139 \mathrm{~F}_{1}+0.040 \mathrm{~F}_{2}-0.143 \mathrm{~F}_{3}+0.038 \mathrm{~F}_{4}+0.140 \mathrm{~F}_{5}- \\
& 0.020 \mathrm{~F}_{6}-0.042 \mathrm{~F}_{7}-0.049 \mathrm{~F}_{8}+0.116 \mathrm{~F}_{9}+0.148 \mathrm{~F}_{10}- \\
& 0.002 \mathrm{~F}_{11}+0.033 \mathrm{~F}_{12}+0.138 \mathrm{~F}_{13}-0.020 \mathrm{~F}_{14} \\
& +0.122 \mathrm{~F}_{15}+0.121 \mathrm{~F}_{16}+0.026 \mathrm{~F}_{17}+0.003 \mathrm{~F}_{18}+0.004 \mathrm{~F} \\
& { }_{19}+0.005 \mathrm{~F}_{20}+0.020 \mathrm{~F}_{21} \\
& \mathrm{~A}_{3}=0.021 \mathrm{~F}_{1}+0.047 \mathrm{~F}_{2}+0.082 \mathrm{~F}_{3}+0.289 \mathrm{~F}_{4}+0.076 \mathrm{~F}_{5}+ \\
& 0.114 \mathrm{~F}_{6}+0.323 \mathrm{~F}_{7}+0.225 \mathrm{~F}_{8}+0.077 \mathrm{~F}_{9}+0.013 \mathrm{~F}_{10}- \\
& 0.022 \mathrm{~F}_{11}-0.302 \mathrm{~F}_{12}-0.095 \mathrm{~F}_{13}-0.107 \mathrm{~F}_{14}-0.035 \mathrm{~F}_{15}+
\end{aligned}
$$


<smiles>c1ccc2cccc-2cc1</smiles><smiles>OCc1ccco1</smiles>

Peak 2<smiles>CC12CCC(CC1)C(C)(C)O2</smiles>

Peak 7<smiles>CC1=CC(=O)CC(C)C1</smiles>

Peak 8<smiles>CC(C)C(=O)CC1(C(C)C)CC1</smiles>

Peak 9<smiles>Cc1ccccc1O</smiles>

Peak 10<smiles>Oc1ccccc1O</smiles>

Peak 12<smiles>C=CCc1ccc(O)c(OC)c1</smiles>

Peak 14<smiles>CCc1ccc(O)cc1</smiles>

Peak 18<smiles>Cc1cc(C(C)(C)C)cc(O)c1O</smiles>

Peak 19

FIgURE 6: The structures of 10 common peaks identified by QTOF/MS.

TABLE 1: Identification of chemical constituents of moxa smoke by UHPLC-QTOF/MS.

\begin{tabular}{|c|c|c|c|c|c|c|c|}
\hline No. & $\begin{array}{c}t_{\mathrm{R}} / \\
\min \end{array}$ & Formula & Parent ion & Theoretical mass $(\mathrm{m} / \mathrm{z})$ & Measured mass $(\mathrm{m} / \mathrm{z})$ & Identification & Ref \\
\hline 1 & 1.55 & $\mathrm{C}_{10} \mathrm{H}_{8}$ & {$[\mathrm{M}-\mathrm{H}]^{-}$} & 128.06 & 128.00 & Azunlene & {$[25]$} \\
\hline 2 & 4.36 & $\mathrm{C}_{5} \mathrm{H}_{4} \mathrm{O}_{2}$ & {$[\mathrm{M}-\mathrm{H}]^{-}$} & 98.09 & 98.92 & Furfurl alcohol & {$[25]$} \\
\hline 3 & 5.36 & $\mathrm{C}_{7} \mathrm{H}_{8} \mathrm{O}$ & {$[\mathrm{M}-\mathrm{H}]^{+}$} & 108.06 & 108.08 & o-Cresol & [25] \\
\hline 4 & 6.84 & $\mathrm{C}_{6} \mathrm{H}_{6} \mathrm{O}_{2}$ & {$[\mathrm{M}-\mathrm{H}]^{+}$} & 110.11 & 110.09 & Catechol & {$[25]$} \\
\hline 5 & 8.12 & $\mathrm{C}_{10} \mathrm{H}_{12} \mathrm{O}_{2}$ & {$[\mathrm{M}-\mathrm{H}]^{+}$} & 164.08 & 164.08 & Eugenol & {$[26]$} \\
\hline 6 & 8.97 & $\mathrm{C}_{8} \mathrm{H}_{12} \mathrm{O}$ & {$[\mathrm{M}-\mathrm{H}]^{+}$} & 124.09 & 124.09 & 3,5-Dimethyl-2-cyclohexen-1-one & [27] \\
\hline 7 & 11.84 & $\mathrm{C}_{11} \mathrm{H}_{16} \mathrm{O}_{2}$ & {$[\mathrm{M}-\mathrm{H}]^{+}$} & 180.11 & 180.09 & $\begin{array}{l}\text { 1,2-Benzenediol,5-(1,1-dimethylethyl)-3- } \\
\text { methyl }\end{array}$ & {$[27]$} \\
\hline 8 & 14.36 & $\mathrm{C}_{10} \mathrm{H}_{16} \mathrm{O}$ & {$[\mathrm{M}-\mathrm{H}]^{+}$} & 152.12 & 152.10 & $-\alpha$-Thujone & {$[26]$} \\
\hline 9 & 24.94 & $\mathrm{C}_{8} \mathrm{H}_{10} \mathrm{O}$ & {$[\mathrm{M}-\mathrm{H}]^{-}$} & 122.07 & 122.03 & 4-Ethylphenol & {$[25]$} \\
\hline 10 & 38.35 & $\mathrm{C}_{10} \mathrm{H}_{18} \mathrm{O}$ & {$[\mathrm{M}-\mathrm{H}]^{+}$} & 154.14 & 154.98 & Cineole & $\begin{array}{l}{[28,} \\
29]\end{array}$ \\
\hline
\end{tabular}

TABLE 2: The similarity results of 12 batches of moxa smoke decoction at $270 \mathrm{~nm}$.

\begin{tabular}{|c|c|c|c|c|c|c|c|c|c|c|c|c|}
\hline No. & $\mathrm{S}_{1}$ & $\mathrm{~S}_{2}$ & $\mathrm{~S}_{3}$ & $\mathrm{~S}_{4}$ & $\mathrm{~S}_{5}$ & $\mathrm{~S}_{6}$ & $\mathrm{~S}_{7}$ & $\mathrm{~S}_{8}$ & $\mathrm{~S}_{9}$ & $\mathrm{~S}_{10}$ & $\mathrm{~S}_{11}$ & $\mathrm{~S}_{12}$ \\
\hline 1 & 1 & & & & & & & & & & & \\
\hline 2 & 0.794 & 1 & & & & & & & & & & \\
\hline 3 & 0.669 & 0.779 & 1 & & & & & & & & & \\
\hline 4 & 0.549 & 0.427 & 0.341 & 1 & & & & & & & & \\
\hline 5 & 0.983 & 0.789 & 0.661 & 0.532 & 1 & & & & & & & \\
\hline 6 & 0.912 & 0.629 & 0.565 & 0.426 & 0.904 & 1 & & & & & & \\
\hline 7 & 0.97 & 0.817 & 0.728 & 0.502 & 0.969 & 0.902 & 1 & & & & & \\
\hline 8 & 0.966 & 0.671 & 0.538 & 0.539 & 0.964 & 0.946 & 0.943 & 1 & & & & \\
\hline 9 & 0.966 & 0.732 & 0.565 & 0.565 & 0.969 & 0.891 & 0.959 & 0.979 & 1 & & & \\
\hline 10 & 0.94 & 0.725 & 0.617 & 0.497 & 0.956 & 0.956 & 0.958 & 0.97 & 0.964 & 1 & & \\
\hline 11 & 0.979 & 0.786 & 0.661 & 0.548 & 0.981 & 0.899 & 0.98 & 0.968 & 0.985 & 0.961 & 1 & \\
\hline 12 & 0.733 & 0.824 & 0.958 & 0.397 & 0.738 & 0.645 & 0.804 & 0.609 & 0.636 & 0.69 & 0.723 & 1 \\
\hline
\end{tabular}

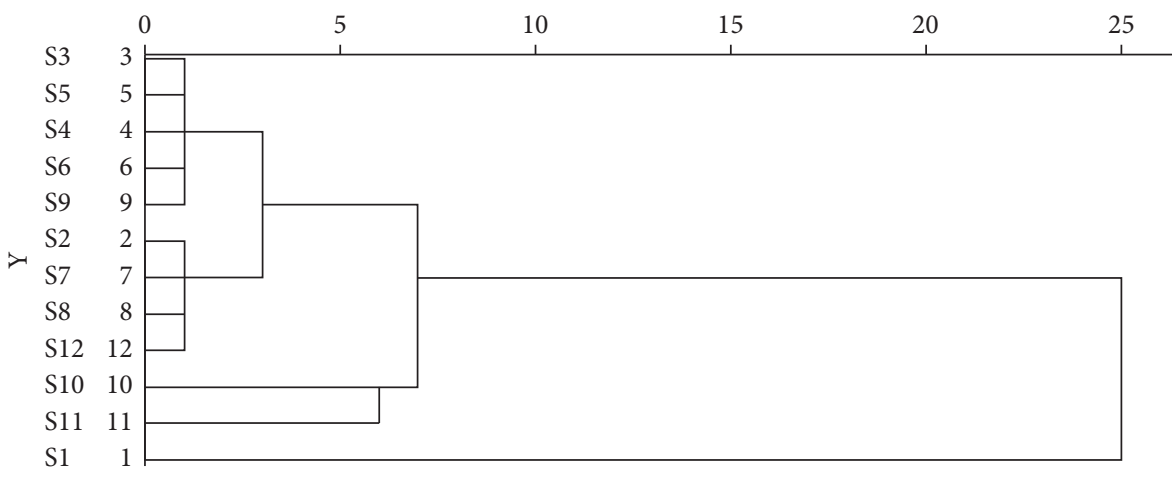

FIgURE 7: Cluster analysis diagram of 12 batches of moxa smoke. 


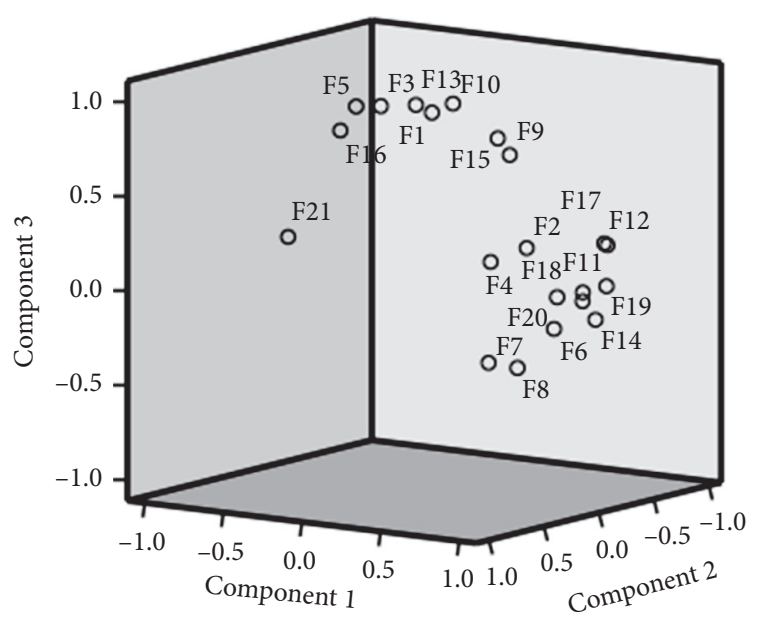

FIgURE 8: Picture of principal component score.

TABle 3: Factor load matrix results.

\begin{tabular}{lccc}
\hline Peak & & Compound & \\
& 1 & 2 & 3 \\
\hline F1 & 0.007 & 0.139 & 0.021 \\
F2 & 0.065 & 0.04 & 0.047 \\
F3 & -0.017 & 0.143 & 0.082 \\
F4 & 0.07 & 0.038 & 0.289 \\
F5 & -0.032 & 0.14 & 0.076 \\
F6 & 0.089 & -0.02 & 0.114 \\
F7 & 0.072 & -0.042 & 0.323 \\
F8 & 0.079 & -0.049 & 0.225 \\
F9 & 0.059 & 0.116 & 0.077 \\
F10 & 0.018 & 0.148 & 0.013 \\
F11 & 0.091 & -0.002 & -0.022 \\
F12 & 0.074 & 0.033 & -0.302 \\
F13 & -0.015 & 0.138 & -0.095 \\
F14 & 0.09 & -0.02 & -0.107 \\
F15 & 0.04 & 0.122 & -0.035 \\
F16 & -0.037 & 0.121 & 0.12 \\
F17 & 0.065 & 0.026 & -0.401 \\
F18 & 0.086 & 0.003 & -0.073 \\
F19 & 0.088 & 0.004 & -0.184 \\
F20 & 0.088 & 0.005 & 0.089 \\
F21 & -0.085 & 0.021 & -0.042 \\
\hline
\end{tabular}

$$
\begin{aligned}
& 0.120 \mathrm{~F}_{16}-0.401 \mathrm{~F}_{17}-0.073 \mathrm{~F}_{18}-0.184 \mathrm{~F}_{19}+0.089 \mathrm{~F}_{20} \\
& -0.042 \mathrm{~F}_{21}
\end{aligned}
$$

Components 1-3 represent 3 principal components, respectively. $F_{1}-F_{21}$ represent normalized relative peak area data for each chromatographic peak. Of the 3 principal components, component 1 is the peak with the largest eigenvalue. The top 5 variable coefficients in the $1^{\text {st }}$ principal component were 0.091, 0.090, 0.089, 0.088, and 0.086.

Peaks 2, 4, 6-9, 11-12, 14, and 17-20 had obvious positive -phase load in the first principal component, indicating that the characteristic value of the $1^{\text {st }}$ principal component increases with the increase of its specific gravity (Table 2). Similarly, peaks $\mathbf{1 ,} \mathbf{3}, \mathbf{5}, \mathbf{9 - 1 0}, \mathbf{1 3}$, and $\mathbf{1 5 - 1 6}$ are the main determinants in the $2^{\text {nd }}$ principal component. In the

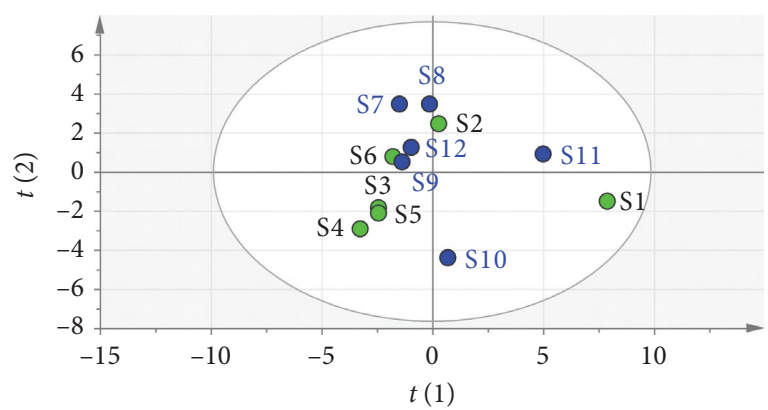

FIGURE 9: OPLS-DA score of 12 batches of moxa smoke samples.

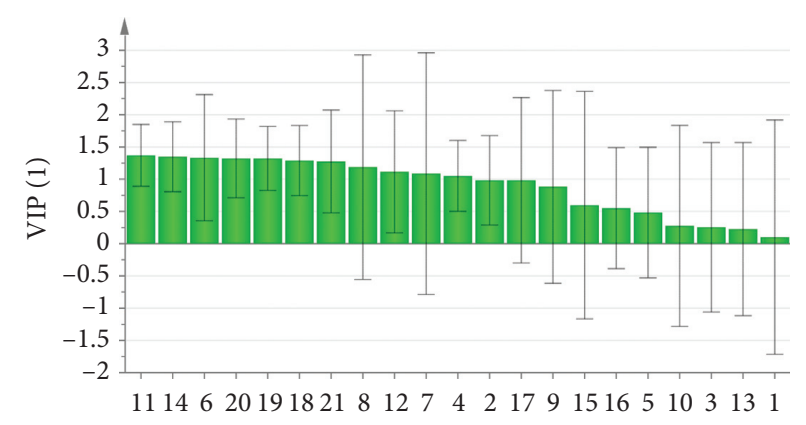

FIgUre 10: The VIP value for classification of common peaks from 12 batches of moxa smoke samples.

$3^{\text {rd }}$ principal component, peaks 3-9, 16, and 20 have obvious positive-phase loads.

3.3.6. Orthogonal Partial Least Squares Discriminant Anal$y$ sis (OPLS-DA). In order to further illustrate the weight of the potential chemical index components in moxa smoke solutions, we utilized OPLS-DA to analyze the variables of the peak area, as shown in Figure 9. The result showed that 12 batches of samples were divided into five categories, which were consistent with the results of clustering analysis. In addition, 21 components were further screened based on the variable importance of the projection (VIP) value for selecting the markers (Figure 10). VIP represents the difference of variables. When the VIP value is greater than 1.0, it means that the component plays a vital role in the differentiation. Based on the OPLS-DA, the VIP values of peaks $11,14,6,20,19,18,21,8,12,7$, and 4 were $>1.0$. The three components, namely, eugenol (peak 14), 4-ethylphenol (peak 18), and 1, 2-benzenediol,5-(1,1-dimethylethyl)-3methyl (peak 19), had a great influence on the differentiation.

\section{Conclusions}

Here, novel UHPLC fingerprints of moxa smoke solutions at a characteristic $270 \mathrm{~nm}$ wavelength were developed for the first time. 21 common peaks in the fingerprint chromatograms were obtained through the similarity evaluation system. Combined with UHPLC-QTOF/MS in both the positive and negative ion mode, 10 common peaks in the 21 common peaks were identified or tentatively characterized 
based on their $t_{\mathrm{R}}$, UV spectra, MS spectra, and previous reports. Similarity, clustering analysis, and principal component analysis showed that mugwort sticks purchased from Henan, Shangdong, Shangxi and Jiangsu, Hunan, Anhui, and Ningxia regions of China have major differentiation. Additionally, pharmacodynamic analysis indicated both moxa smoke and its aqueous solution may significantly promote healing of wounds with superficial infection. Our data indicate that because its main effects are unchanged, transformation of moxa smoke into aqueous moxa smoke not only reduces harm but also is a simple and controllable procedure. Importantly, our findings offer reference for clinical applications.

\section{Data Availability}

The data used to support the findings of this study are available from the corresponding author upon request.

\section{Conflicts of Interest}

The authors declare that there are no conflicts of interest.

\section{Authors' Contributions}

Yanling Wang and Shengbing $\mathrm{Wu}$ contributed equally to this work.

\section{Acknowledgments}

The authors are grateful to the Key Research and Development Plan Projects of Anhui Province (1804b06020353) and Anhui TCM Leading Talents Construction Project (ZYYLJRC201911). This work was supported by the Natural Science Foundation of Anhui Province (2008085MH271) and Talent support project of Anhui University of Chinese Medicine (2019rczd002).

\section{References}

[1] Y. Jiang, B. David, P. Tu, and Y. Barbin, "Recent analytical approaches in quality control of traditional Chinese medicines-A review," Analytica Chimica Acta, vol. 657, no. 1, pp. 9-18, 2010.

[2] D. Y. W. Lee, Q. Y. Li, J. Liu, and T. Efferth, "Traditional Chinese herbal medicine at the forefront battle against COVID-19: clinical experience and scientific basis," Phytomedicine, vol. 80, Article ID 153337, 2021.

[3] S. Shen, M. Wang, and J. Dong, "Moxibustion therapy for chronic spontaneous urticaria," Medicine, vol. 99, no. 46, Article ID e23226, 2020.

[4] X. Li, G. Guo, F. Shen et al., "Moxibustion activates macrophage autophagy and protects experimental mice against bacterial infection," Evidence-Based Complementary and Alternative Medicine, vol. 2014, Article ID 450623, 6 pages, 2014.

[5] C. Zhou, X. Feng, J. Wang et al., "Research advance on moxa smoke," Journal of Acupuncture and Tuina Science, vol. 9, no. 2, pp. 67-72, 2011.

[6] C. Yu, N. Zhang, W. Zhu et al., "Does moxa smoke have significant effect on the acupuncturist's respiratory system? a population-based study," Evidence-Based Complementary and
Alternative Medicine, vol. 2019, Article ID 4873235, 10 pages, 2019.

[7] Y. Cui, B. Zhao, Y. Huang et al., "Effects of moxa (Folium artemisiae argyi) smoke exposure on heart rate and heart rate variability in healthy young adults: a randomized, controlled human study," Evidence-Based Complementary and Alternative Medicine, vol. 2013, Article ID 510318, 10 pages, 2013.

[8] J. Huang, Y.-H. Huang, M. Y. Lim et al., "Emission characteristics and concentrations of gaseous pollutants in environmental moxa smoke," Aerosol and Air Quality Research, vol. 16, no. 2, pp. 398-404, 2016.

[9] L. Wang, L. Han, P. Liu et al., "Moxa smoke: is it a beneficial or hazardous factor for infertility? a preclinical study on sperm parameters and sex hormones in male rats," European Journal of Integrative Medicine, vol. 8, no. 1, pp. 28-35, 2016.

[10] T. Matsumoto, S. Katai, and T. Namiki, "Safety of smoke generated by Japanese moxa upon combustion," European Journal of Integrative Medicine, vol. 8, no. 4, pp. 414-422, 2016.

[11] X. Xu, S. Shan, W. Wang, and H. Liu, "Analysis of the components in moxa smoke by GC-MS and preliminary discussion on its toxicity and side effects," Evidence-based Complementary and Alternative Medicine, vol. 2020, Article ID 2648759, 13 pages, 2020.

[12] Y. Zhang, L. Kang, H. Li et al., "Characterization of moxa floss combustion by TG/DSC, TG-FTIR and IR," Bioresource Technology, vol. 288, Article ID 121516, 2019.

[13] Q. Li, H. Guan, X. Wang et al., "Fingerprint-efficacy study of the quaternary alkaloids in corydalis yanhusuo," Journal of Ethnopharmacology, vol. 207, pp. 108-117, 2017.

[14] M. Liao, H. Shang, Y. Li et al., "An integrated approach to uncover quality marker underlying the effects of Alisma orientale on lipid metabolism, using chemical analysis and network pharmacology," Phytomedicine, vol. 45, pp. 93-104, 2018.

[15] A. Zhou, Q. Xu, W. J. Tang et al., “A comparative analysis of chemical components in volatile oil and smoke of moxa stick by gas chromatography-mass spectroscopy," Journal of Anhui University of Chinese Medicine, vol. 35, pp. 80-82, 2016.

[16] G. X. Shi, T. M. Wang, S. B. Wu et al., "Activity of essential oil extracted from Artemisia argyi in inducing apoptosis of Candida albicans," China Journal of Chinese Materia Medica, vol. 42, pp. 3572-3577, 2017.

[17] M. Liu, J. Zhu, S. Wu et al., "De novo assembly and analysis of the Artemisia argyi transcriptome and identification of genes involved in terpenoid biosynthesis," Scientific Reports, vol. 8, no. 1, p. 5824, 2018.

[18] Z. Song, F. Shen, Z. Zhang, S. Wu, and G. Zhu, "Calpain inhibition ameliorates depression-like behaviors by reducing inflammation and promoting synaptic protein expression in the hippocampus," Neuropharmacology, vol. 174, Article ID 108175, 2020.

[19] L. Wang, Y. Wang, G. Tong et al., "Development of a novel UHPLC-UV combined with UHPLC-QTOF/MS fingerprint method for the comprehensive evaluation of Nao-Luo-XinTong: multi-wavelength setting based on traditional Chinese medicinal prescription composition," Analytical Methods, vol. 11, no. 48, pp. 6092-6102, 2019.

[20] L. Shao, C. Sun, W. Lu et al., "Effects of borneol on the release of compound danshen colon-specific osmotic pump capsule in vitro and pharmacokinetics study in beagle dogs," AAPS PharmSciTech, vol. 21, no. 8, p. 316, 2020.

[21] B. Wang, X. Liu, A. Zhou, M. Meng, and Q. Li, "Simultaneous analysis of coumarin derivatives in extracts of Radix Angelicae 
pubescentis (Duhuo) by HPLC-DAD-ESI-MSn technique," Analytical Methods, vol. 6, no. 19, pp. 7996-8002, 2014.

[22] Z. H. Fang, Z. J. Yang, X. P. Zeng et al., "Establishment of skin woud infection model in rats," Medical Journal of Wuhan University, vol. 39, pp. 412-416, 2018.

[23] S. Yu, L. Zhu, Z. Xiao et al., "Rapid fingerprint analysis of flos carthami by ultra-performance liquid chromatography and similarity evaluation," Journal of Chromatographic Science, vol. 54, no. 9, pp. 1619-1624, 2016.

[24] C. Zhao, X. Qiao, Q. Shao, M. Hassan, and Z. Ma, "Evolution of the lignin chemical structure during the bioethanol production process and its inhibition to enzymatic hydrolysis," Energy \& Fuels, vol. 34, no. 5, pp. 5938-5947, 2020.

[25] R. Jin, B. X. Zhao, M. M. Yu et al., "Qualitative analysis on components of moxa combustion products by solid-phase microextraction-gas chromatography-mass spectrography," Journal of Beijing University of Traditional Chinese Medicine, vol. 34, pp. 632-636, 2011.

[26] K. Zheng, X. F. Zhong, and H. Zhang, "Advances in Research on constituents and pharmacological effects of Artemisia argyi essential oil," Chinese Journal of Experimental Traditional Medical Formulae, vol. 26, pp. 224-234, 2020.

[27] M. Yang, D. Jiang, Y. Yi, and Z. Hong, "Observation on scavenging free radical function of Artemisia burning products," Chinese Acupuncture, vol. 29, no. 7, pp. 547-549, 2009.

[28] U. R. Juergens, U. Dethlefsen, G. Steinkamp, A. Gillissen, R. Repges, and H. Vetter, "Anti-inflammatory activity of 1.8cineol (eucalyptol) in bronchial asthma: a double-blind placebo-controlled trial," Respiratory Medicine, vol. 97, no. 3, pp. 250-256, 2003.

[29] A. Abdul, A. Mohammed, K. Kanchan et al., "Role of novel terpenes in transcutaneous permeation of valsartan: effectiveness and mechanism of action," Drug Development and Industrial Pharmacy, vol. 37, pp. 583-596, 2011. 